THE ASTROPHYSICAL JournaL, 344:311-319, 1989 September 1

(C) 1989. The American Astronomical Society. All rights reserved. Printed in U.S.A.

\title{
THE ABUNDANCES OF ATOMIC CARBON AND CARBON MONOXIDE COMPARED WITH VISUAL EXTINCTION IN THE OPHIUCHUS MOLECULAR CLOUD COMPLEX
}

\author{
MARgaret A. Frerking \\ Jet Propulsion Laboratory, California Institute of Technology \\ AND \\ Jocelyn Keene, Geoffrey A. Blake, And T. G. Phillips \\ California Institute of Technology \\ Received 1985 March 11; accepted 1989 February 1
}

\begin{abstract}
We have observed emission from the $492 \mathrm{GHz}$ lines of $\mathrm{C}$ I toward six positions in the Ophiuchus molecular cloud complex for which accurate visual extinctions are available. We find that the column density of $\mathrm{C}_{\mathrm{I}}$ increases with $A_{V}$ to greater than $2 \times 10^{17} \mathrm{~cm}^{-2}$ at $100 \mathrm{mag}$, the column-averaged fractional abundance reaches a peak of about $2.2 \times 10^{-5}$ for $A_{V}$ in the range 4-11 mag and the column-averaged abundance ratio of $\mathrm{C}_{\mathrm{I}}$ to $\mathrm{CO}$ decreases with $A_{V}$ from about 1 at $2 \mathrm{mag}$ to greater than $\sim 0.03$ at $100 \mathrm{mag}$. These results imply that, while $\mathrm{C}_{\mathrm{I}}$ is not the primary reservoir of gaseous carbon even at cloud edges, its fractional abundance remains high for at least $10 \mathrm{mag}$ into the cloud and may be significant at even greater depths.
\end{abstract}

Subject headings: interstellar: matter — interstellar: molecules

\section{INTRODUCTION}

Carbon is an important constituent of the interstellar medium; its cosmic abundance is comparable to that of nitrogen and oxygen. Chemical theories of molecular cloud composition (e.g., Langer 1976; Mitchell, Ginsburg, and Kuntz 1978; Iglesias 1977; Prasad and Huntress 1980; de Jong, Dalgarno, and Boland 1980; Boland and de Jong 1984; Glassgold, Huggins, and Langer 1985; Tielens and Hollenbach 1985) predict that in the diffuse interstellar medium carbon is primarily in the form of ionized carbon (C II); near the surface of molecular clouds in the form of atomic carbon (C I); and in cloud interiors in the form of carbon monoxide (CO). In contrast to these theories, observations (Phillips and Huggins 1981; Wootten et al. 1982; Keene et al. 1985) suggest that the C I abundance remains large even deep within clouds. Several explanations for these observations have been suggested and have recently been reviewed by Keene et al. (1985). We will compare our observations with two recent calculations of cloud abundances. The first is a study of equilibrium chemistry in a variety of different cloud models by van Dischoeck and Black (1988). They incorporated into their calculations the effects of variations in the incident UV radiation field and in the depletion of gas-phase carbon. The second is a study of nonequilibrium chemistry in physically evolving clouds by Tarafdar et al. (1985). A third possibility recently put forth by Stutzki et al. (1988) and Genzel et al. (1988), but not considered quantitatively herein, is that the cloud structures are clumpy and are transparent to UV radiation, either interstellar UV or UV generated locally by embedded stars.

In order to determine the relationships among $\mathrm{C}_{\mathrm{I}}$ abundance, $\mathrm{CO}$ abundance, and visual extinction (hence $\mathrm{H}_{2}$ abundance), we have observed the $492 \mathrm{GHz}$ line of $\mathrm{C}$ I toward several positions in the Ophiuchus molecular cloud complex for which accurate visual extinctions (Elias 1978) and CO abundances (Frerking, Langer, and Wilson 1982, hereafter FLW) are available. The range of visual extinctions observed is from 2.6 to $100 \mathrm{mag}$. This data set complements those of
Jenkins and Shaya (1979) and Federman et al. (1980) which include a range of 0.1 to $1.5 \mathrm{mag}$ extinction.

\section{OBSERVATIONS}

Emission from the ${ }^{3} P_{1} \rightarrow{ }^{3} P_{0}$ transition of $\mathrm{C}$ I has been observed toward six positions in the Ophiuchus molecular cloud complex (Table 1 ). In addition, the $J=1 \rightarrow 0$ lines of $\mathrm{C}^{18} \mathrm{O},{ }^{13} \mathrm{CO}$, and ${ }^{12} \mathrm{CO}$ were observed for the two highest extinction sources since they were not included in the FLW study.

The C I observations were carried out in 1981 and 1983 August, and in 1984 June with the $0.9 \mathrm{~m}$ diameter telescope of the NASA Kuiper Airborne Observatory at an altitude of 12.5 $\mathrm{km}$, above most of the terrestrial water vapor that causes the atmosphere to be opaque from sea level at submillimeter wavelengths. The receiver was the single-channel InSb heterodyne bolometer system described by Brown, Keene, and Phillips (1985). For the four lower extinction sources, a single $0.3 \mathrm{~km}$ $\mathrm{s}^{-1}$ wide channel was swept across the spectral range of interest by stepping the local oscillator frequency. A $0.6 \mathrm{~km} \mathrm{~s}^{-1}$ wide channel stepped at $0.5 \mathrm{~km} \mathrm{~s}^{-1}$ intervals was used for the two sources at high visual extinction. The system noise temperature, determined by observation of ambient and liquid nitrogen temperature loads, was about $580 \mathrm{~K}$ in 1981 and $320 \mathrm{~K}$ in 1983 and 1984 . The diffraction-limited beam size for the $C_{\text {I }}$ measurements was 2.7 ; the beam efficiency derived from observations of the moon was $35 \%$ for the 1981 flights and $50 \%$ for the 1983 flights. We were unable to measure the beam efficiency in the 1984 flight; however, based on a previous flight in the same series, we estimate that it was also $50 \%$. One of the positions, R35, was observed in both 1981 and 1983 to confirm the instrument calibration. Similarly, Oph A was observed in both 1983 and 1984. The calibrations from the different flights agreed to within $15 \%$. The spectra were obtained by position switching between the source position and two symmetrically located reference positions chosen to be 
TABLE 1

\begin{tabular}{|c|c|c|}
\hline \multicolumn{3}{|c|}{ SOURCE COORDINATES } \\
\hline Source & R.A. (1950) & Decl. (1950) \\
\hline R17 & $16^{\mathrm{h}} 23^{\mathrm{m}} 11^{\mathrm{s}} 6$ & $-23^{\circ} 11^{\prime} 54^{\prime \prime}$ \\
\hline Oph A & 162325.0 & -241549 \\
\hline Oph B & 162409.9 & -242242 \\
\hline R35 ... & 162445.2 & -241643 \\
\hline R38 & $\begin{array}{lll}16 & 25 & 07.8\end{array}$ & -251644 \\
\hline R48 $\ldots \ldots \ldots \ldots$ & 163648.9 & -240019 \\
\hline
\end{tabular}

regions of low optical obscuration on the Palomar Sky Survey prints.

The CO observations for Oph A and Oph B were carried out in 1984 September using the Millimeter Wave Observatory $4.9 \mathrm{~m}$ antenna at Fort Davis, Texas, ${ }^{1}$ with a velocity resolution of $0.17 \mathrm{~km} \mathrm{~s}^{-1}$. The diffraction-limited beam size at $115 \mathrm{GHz}$ was 2.3 , similar to that for the $\mathrm{C}_{\mathrm{I}}$ observations. The beam efficiency was assumed to be $85 \%$.

Positive detections of the $\mathrm{C}_{\mathrm{I}}$ line were obtained for five of the six source locations. A linear baseline was removed from all the spectra, and they were scaled to $T_{A}{ }^{*}$ (also called $T_{R}{ }^{*}$ by Kutner and Ulich 1981) by dividing by the beam efficiency. Table 2 gives the line parameters for each source, including $V_{P}$, the velocity of the peak line intensity, $T_{A}{ }^{*}$, the peak antenna temperature, $\Delta V$, the half power full velocity width of the line, and the integrated area under the line.

\section{RESULTS}

a) Line Frequency for the ${ }^{3} P_{1} \rightarrow{ }^{3} P_{0}$ Transition

At the time these observations were made, the rest frequency of the ${ }^{3} P_{1} \rightarrow{ }^{3} P_{0}$ transition of $C_{\mathrm{I}}$ had been measured in the

\footnotetext{
${ }^{1}$ The Millimeter Wave Observatory is operated by the Electrical Engineering Research Laboratory of the University of Texas at Austin, with support from the National Science Foundation and McDonald Observatory.
}

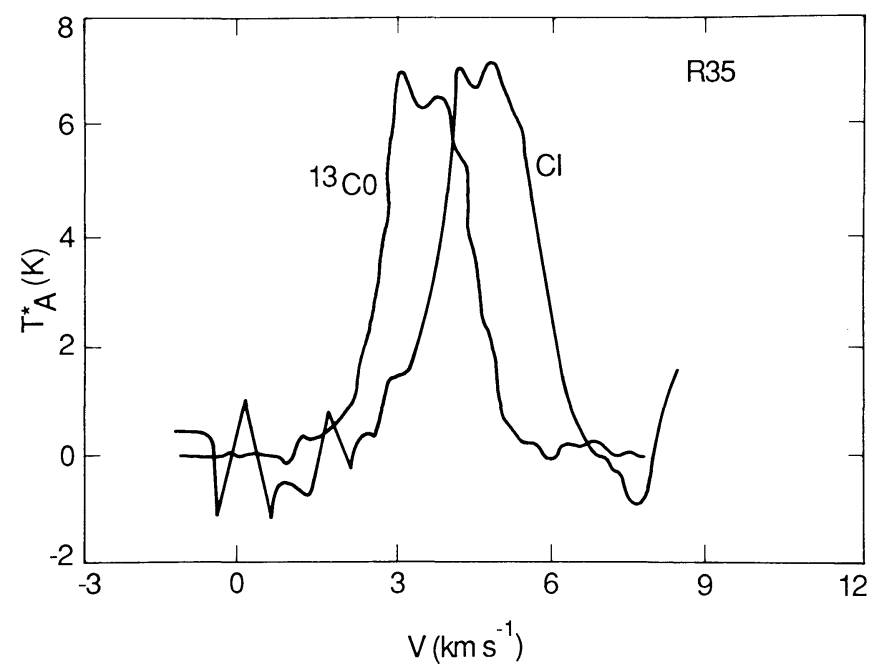

FIG. 1.-The $\mathrm{C}_{\mathrm{I}}$ and ${ }^{13} \mathrm{CO}$ lines toward R35. The line shapes are similar but shifted with respect to each other by $1.64 \mathrm{MHz}$. Based on this observation

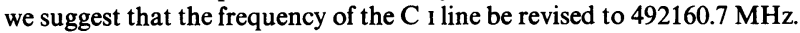

laboratory by LMR spectroscopy to be $492162.3 \pm 0.9 \mathrm{MHz}$ (Saykally and Evanson 1980). The observed spectrum for the R35 position, taken in 1981, is shown in Figure 1 along with the ${ }^{13} \mathrm{CO}(J=1 \rightarrow 0)$ spectrum for the same position. The two spectra have essentially the same shape but are displaced from each other by $1.64 \mathrm{MHz}$ in frequency. The similarity of the spectral shapes suggests that emission in the two species arises under similar conditions. Since the frequency of the ${ }^{13} \mathrm{CO}$ transition is well determined from numerous radio-astronomical observations, it appears that the frequency of the $\mathrm{C}_{\mathrm{I}}$ line should be modified to $492160.7 \pm 0.1 \mathrm{MHz}$. This frequency offset is consistent with detailed comparisons of the $\mathrm{C}_{\mathrm{I}}$ and CO line profiles in other sources such as M17 and S140 (Keene et al. 1985). More recent laboratory measurements and re-

TABLE 2

Line PARAmeters

\begin{tabular}{|c|c|c|c|c|c|c|}
\hline Source & $\begin{array}{c}A_{V}{ }^{\mathrm{a}} \\
(\mathrm{mg})\end{array}$ & $\begin{array}{c}V_{P}\left(\mathrm{C}^{18} \mathrm{O}\right)^{\mathrm{b}} \\
\left(\mathrm{km} \mathrm{s}^{-1}\right)\end{array}$ & $\begin{array}{c}\mathrm{C} \mathrm{I} \\
T_{A}^{*}(\mathrm{~K}) \\
\Delta V\left(\mathrm{~km} \mathrm{~s}^{-1}\right) \\
\text { Area }\left(\mathrm{K} \mathrm{km} \mathrm{s}^{-1}\right)\end{array}$ & $\begin{array}{c}{ }^{12} \mathrm{CO}^{\mathrm{b}} \\
T_{A}^{*}(K) \\
\Delta V\left(\mathrm{~km} \mathrm{~s}^{-1}\right) \\
\text { Area }\left(\mathrm{K} \mathrm{km} \mathrm{s}^{-1}\right)\end{array}$ & $\begin{array}{c}{ }^{13} \mathrm{CO}^{\mathrm{b}} \\
T_{A}^{*}(\mathrm{~K}) \\
\Delta V\left(\mathrm{~km} \mathrm{~s}^{-1}\right) \\
\text { Area }\left(\mathrm{K} \mathrm{km} \mathrm{s}^{-1}\right)\end{array}$ & $\begin{array}{c}\mathrm{C}^{18} \mathrm{O}^{\mathrm{b}} \\
T_{A}^{*}(\mathrm{~K}) \\
\Delta V\left(\mathrm{~km} \mathrm{~s}^{-1}\right) \\
\text { Area }\left(\mathrm{K} \mathrm{km} \mathrm{s}^{-1}\right)\end{array}$ \\
\hline R17 ........... & 2.6 & $2.71^{\mathrm{c}}$ & $<2^{d}$ & $\begin{array}{l}9.8 \pm 0.6 \\
0.8 \pm 0.1\end{array}$ & $\begin{array}{l}0.43 \pm 0.09 \\
0.53 \pm 0.13\end{array}$ & \\
\hline & & & $<0.6^{\mathrm{d}}$ & $8.9 \pm 0.7$ & $0.24 \pm 0.09$ & $<0.05$ \\
\hline R48 $\ldots \ldots \ldots$ & 4.4 & 4.96 & $\begin{array}{l}3.0 \pm 0.7 \\
0.9 \pm 0.2 \\
4.1 \pm 0.3\end{array}$ & $\begin{array}{r}12.3 \pm 0.6 \\
1.9 \pm 0.1 \\
23.8+0.7\end{array}$ & $\begin{array}{l}3.77 \pm 0.11 \\
0.79 \pm 0.03 \\
3.48 \pm 0.13\end{array}$ & $\begin{array}{l}0.48 \pm 0.07 \\
0.49 \pm 0.07 \\
0.15+0.06\end{array}$ \\
\hline R38 $\ldots \ldots \ldots$ & 7.1 & 3.28 & $\begin{array}{l}4.5 \pm 1.0 \\
1.8 \pm 0.2 \\
8.0 \pm 0.3\end{array}$ & $\begin{array}{r}16.6 \pm 0.6 \\
2.4 \pm 0.1 \\
40.8 \pm 0.7\end{array}$ & $\begin{array}{r}9.19 \pm 0.18 \\
1.09 \pm 0.02 \\
10.96 \pm 0.24\end{array}$ & $\begin{array}{l}0.56 \pm 0.06 \\
0.97 \pm 0.12 \\
0.49 \pm 0.08\end{array}$ \\
\hline R35 $\ldots \ldots \ldots$ & 11.1 & 3.54 & $\begin{array}{r}5.5 \pm 0.4 \\
2.0 \pm 0.2 \\
12.0 \pm 0.2\end{array}$ & $\begin{array}{r}16.7 \pm 0.6 \\
3.2 \pm 0.1 \\
55.3 \pm 0.7\end{array}$ & $\begin{array}{r}7.16 \pm 0.08 \\
1.82 \pm 0.02 \\
13.82 \pm 0.13\end{array}$ & $\begin{array}{l}0.97 \pm 0.10 \\
1.36 \pm 0.14 \\
1.24 \pm 0.15\end{array}$ \\
\hline Oph A ....... & 80 & 3.2 & $\begin{array}{r}9.9 \pm 0.3 \\
2.2 \pm 0.2 \\
25.6 \pm 0.3\end{array}$ & $\begin{array}{c}32 \ldots \\
\ldots\end{array}$ & $\begin{array}{r}20 \pm 0.2 \\
1.7 \pm 0.2 \\
39.6 \pm 0.3\end{array}$ & $\begin{array}{l}4.6 \pm 0.2 \\
1.2 \pm 0.2 \\
6.2 \pm 0.1\end{array}$ \\
\hline Oph B $\ldots \ldots$ & 101 & 3.5 & $\begin{array}{r}5.5 \pm 0.5 \\
2.5 \pm 0.3 \\
16.4 \pm 0.3\end{array}$ & $\begin{array}{c}27 \ldots \\
\ldots \\
\ldots\end{array}$ & $\begin{array}{r}12.5 \pm 0.2 \\
2.4 \pm 0.4 \\
27.6 \pm 0.4\end{array}$ & $\begin{array}{l}5.2 \pm 0.2 \\
1.5 \pm 0.2 \\
8.8 \pm 0.1\end{array}$ \\
\hline
\end{tabular}

${ }^{a}$ From Elias 1978, except for Oph A and Oph B which were calculated from $N\left(\mathrm{C}^{18} \mathrm{O}\right)$ following FLW.

b From FLW except for Oph A and Oph B.

${ }^{c} V_{P}$ determined from ${ }^{13} \mathrm{CO}$ line profile.

d $2 \sigma$ value. 
analysis of the earlier data have led to a revised laboratory frequency of $492161.1 \pm 1.2 \mathrm{MHz}$ (Cooksy et al. 1986), which agrees with the astronomical measurements to within the errors. Henceforth in this paper we use our astronomically determined frequency.

\section{b) Qualitative Comparisons of $\mathrm{C}$ I with $A_{V}$ and $\mathrm{CO}$}

The $\mathrm{C}$ I spectra are shown in Figure 2 along with the corresponding ${ }^{13} \mathrm{CO}(J=1 \rightarrow 0)$ spectra. The qualitative relationships among $\mathrm{C}_{\mathrm{I}}$ abundance, visual extinction, and $\mathrm{CO}$ abundance are evident in the observed spectra and in the line parameters in Table 2.

The $\mathrm{C}_{\mathrm{I}}$ and ${ }^{13} \mathrm{CO}$ line shapes resemble each other in the four highest extinction sources. In R48, the source with the lowest extinction in which $\mathrm{C}_{\mathrm{I}}$ was detected, the $\mathrm{C}_{\mathrm{I}}$ line shape is considerably different from the ${ }^{13} \mathrm{CO}$ line shape, having a larger antenna temperature at low velocity and a lower antenna temperature at high velocity. Interestingly, the lowvelocity wing is also present in the ${ }^{12} \mathrm{CO}$ spectrum (FLW, not shown) suggesting that the ratios of $\mathrm{C}$ I to ${ }^{13} \mathrm{CO}$ and ${ }^{12} \mathrm{CO}$ to ${ }^{13} \mathrm{CO}$ abundances vary along the line of sight toward $\mathrm{R} 48$ but that the $\mathrm{C}$ i to ${ }^{12} \mathrm{CO}$ ratio may be more nearly constant.

For five of the sources the integrated $\mathrm{C}_{\mathrm{I}}$ line intensity increases with $A_{V}$. In the sixth source, Oph B, which has the highest visual extinction [determined from $\int T_{A}{ }^{*}\left(C^{18} \mathrm{O}\right) d V$ as by $\mathrm{FLW}$ ], the $\mathrm{C}_{\mathrm{I}}$ line intensity is smaller than in Oph $\mathrm{A}$. However, both the ${ }^{12} \mathrm{CO}$ and ${ }^{13} \mathrm{CO}$ lines also have smaller integrated intensities in Oph B than they do in Oph A; the ${ }^{12} \mathrm{CO}$ line is strongly self-absorbed in both sources, as is the ${ }^{13} \mathrm{CO}$ line in Oph B. Moreover, the $\mathrm{C}_{\mathrm{I}}$ line in Oph B is quite flat-topped, indicating that probably it is also self-absorbed and its intensity consequently underestimated. The measured antenna temperature ratio, $\mathrm{C}_{\mathrm{I}}$ to ${ }^{13} \mathrm{CO}$, is highest in the intermediate $A_{V}$ source, $\mathrm{R} 35$.

\section{c) Quantitative Comparisons of $\mathrm{C} \mathrm{I}$ and $A_{V}$}

We derive the column density of $\mathrm{C}$ I along the line of sight, $N\left(\mathrm{C}_{\mathrm{I}}\right)$, and the column-averaged fractional $\mathrm{C}_{\mathrm{I}}$ abundance, $X(\mathrm{C} \mathrm{I})=N(\mathrm{C} \mathrm{I}) / N\left(\mathrm{H}_{2}\right)$ as follows. Under the assumption of local thermodynamic equilibrium, the observed line intensity is a function of two parameters: optical depth, $\tau$, and excitation temperature, $T_{\mathrm{ex}}$. This can be seen in the equation of radiative transfer,

$$
T_{A}^{*}=\left(1-e^{-\tau}\right) T_{10}\left(\frac{1}{\exp \left(T_{10} / T_{\mathrm{ex}}\right)-1}-\frac{1}{\exp \left(T_{10} / 2.7\right)-1}\right) .
$$

Here $T_{10}\left(\equiv h v_{10} / k=23.6 \mathrm{~K}\right)$ is the ${ }^{3} P_{1} \rightarrow{ }^{3} P_{0}$ transition temperature of $\mathrm{C}$ I. The excitation temperature is derived from the
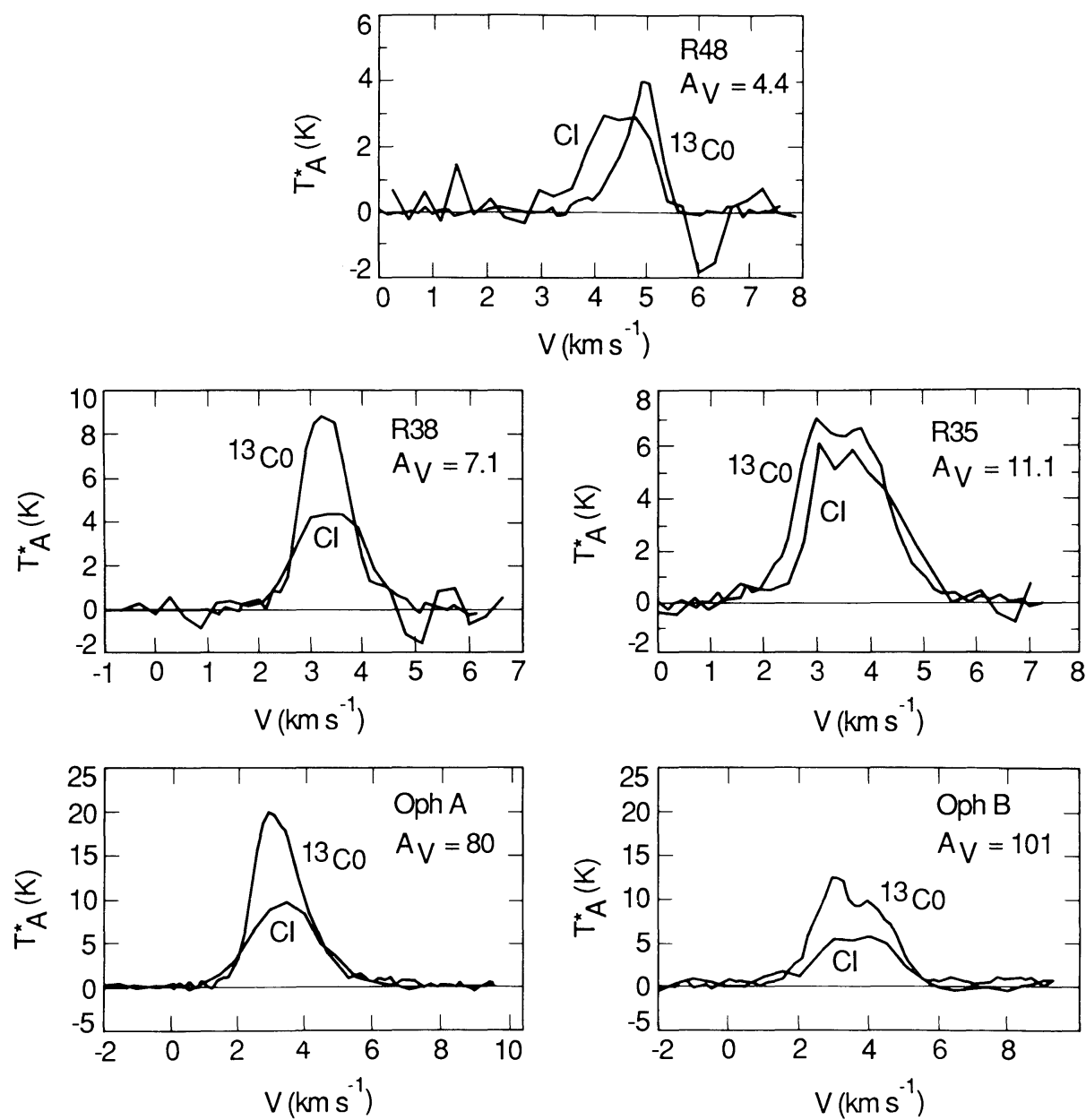

FIG. 2.-The ${ }^{13} \mathrm{CO}$ and $\mathrm{C} \mathrm{I}$ lines for the five positions in which $\mathrm{C} \mathrm{I}$ emission is detected. The frequency of the $\mathrm{C} \mathrm{I}$ lines have been shifted by $1.64 \mathrm{MHz}$ as discussed in the text. 
TABLE 3

Column Densities

\begin{tabular}{|c|c|c|c|c|c|c|}
\hline Source & $A_{V}$ & $\begin{array}{l}T_{\text {ex }} \\
(\mathrm{K})\end{array}$ & $\tau_{0}\left(C_{I}\right)$ & $\begin{array}{c}N(\mathrm{C} \mathrm{I}) \\
\left(10^{16} \mathrm{~cm}^{-2}\right)\end{array}$ & $\begin{array}{c}N\left({ }^{13} \mathrm{CO}\right) \\
\left(10^{16} \mathrm{~cm}^{-2}\right)\end{array}$ & $\begin{array}{c}N\left(\mathrm{C}^{18} \mathrm{O}\right) \\
\left(10^{14} \mathrm{~cm}^{-2}\right)\end{array}$ \\
\hline $\mathbf{R} 17$ & 2.6 & 13.1 & $\ldots$ & $<1.5^{\mathrm{a}}$ & 0.03 & $<0.5$ \\
\hline R48 $\ldots$ & 4.4 & 15.6 & 0.6 & $8.3 \pm 0.7$ & 0.48 & 2.0 \\
\hline R38 ......... & 7.1 & 20.0 & 0.6 & $14.5 \pm 0.6$ & 2.29 & 5.4 \\
\hline R35 $\ldots \ldots \ldots$ & 11.1 & 20.0 & 0.8 & $23.2 \pm 0.7$ & 2.68 & 17.4 \\
\hline Oph A $\ldots \ldots$ & 80 & 35.5 & 0.5 & $43.0 \pm 0.6$ & 11.9 & 129.0 \\
\hline Oph B ...... & 101 & 30.0 & 0.3 & $25.0 \pm 0.5$ & 6.3 & 165.0 \\
\hline
\end{tabular}

a $2 \sigma$ value.

${ }^{12} \mathrm{CO}(J=1 \rightarrow 0)$ peak antenna temperature by assuming that the ${ }^{12} \mathrm{CO}$ emission is optically thick. The $\mathrm{C}_{\mathrm{I}}$ optical depth at line center, $\tau_{0}$ is then derived from the measured $C_{I}$ peak antenna temperature by using equation (1). For the observed sources it ranges from about 0.2 to 0.8 . The column density of the $J=1$ state, $N_{1}$, is related to the optical depth by

$$
N_{1}=\frac{8 \pi v^{2}}{c^{2} A_{10}} \int \tau d v .
$$

In the optically thin limit $\int \tau d v$ is proportional to $\int T_{A}^{*} d V$, so that

$$
N_{1}=5.94 \times 10^{15} \int T_{A}^{*} d V\left(\mathrm{~cm}^{-2}\right),
$$

where an Einstein $A_{10}$ value of $7.9 \times 10^{-8} \mathrm{~s}^{-1}$ has been used (Nussbaumer 1971; Nussbaumer and Rusca 1979). To correct for line saturation we multiply $N_{1}$ by $\tau_{0} /\left(1-e^{-\tau_{0}}\right)$. The total column density, $N(\mathrm{C} \mathrm{I})$, is calculated from $\mathrm{N}_{1}$ by assuming that the $J=2$ state is characterized by the same excitation temperature as $J=1$. That is,

$$
N\left(\mathrm{C}_{\mathrm{I}}\right)=N_{1}\left(e^{23.6 / T_{\mathrm{ex}}}+3+5 e^{-38.8 / T_{\mathrm{ex}}}\right) / 3 .
$$

For the two sources at high $A_{V}$, Oph A and Oph B, the optical depth is probably underestimated due to self-absorption, thus $N(\mathrm{C}$ I) may be considerably greater than calculated.

The resulting values for $N(\mathrm{C} \mathrm{I})$ are listed in Table 3 and plotted in Figure 3 as a function of $A_{V}$, along with the column densities from Jenkins and Shaya (1979) at lower $A_{V}$. The visual extinctions for Oph $\mathrm{A}$ and $\mathrm{Oph} \mathrm{B}$ were obtained from the $\mathrm{C}^{18} \mathrm{O}$ column densities as described by FLW since infrared measurements are not available. The observed column density of $C_{I}$ increases from a visual extinction of $0.1 \mathrm{mag}$ to $10 \mathrm{mag}$. Above $10 \mathrm{mag}$ it appears to saturate at $4 \times 10^{17} \mathrm{~cm}^{-2}$. The rate of increase steepens sharply at about $1 \mathrm{mag}$, indicative of a change in chemical processes at the cloud edge.

In Figure 4 the column-averaged fractional abundance of C I, $N(\mathrm{C} \mathrm{I}) / N\left(\mathrm{H}_{2}\right)$, is shown as a function of visual extinction,

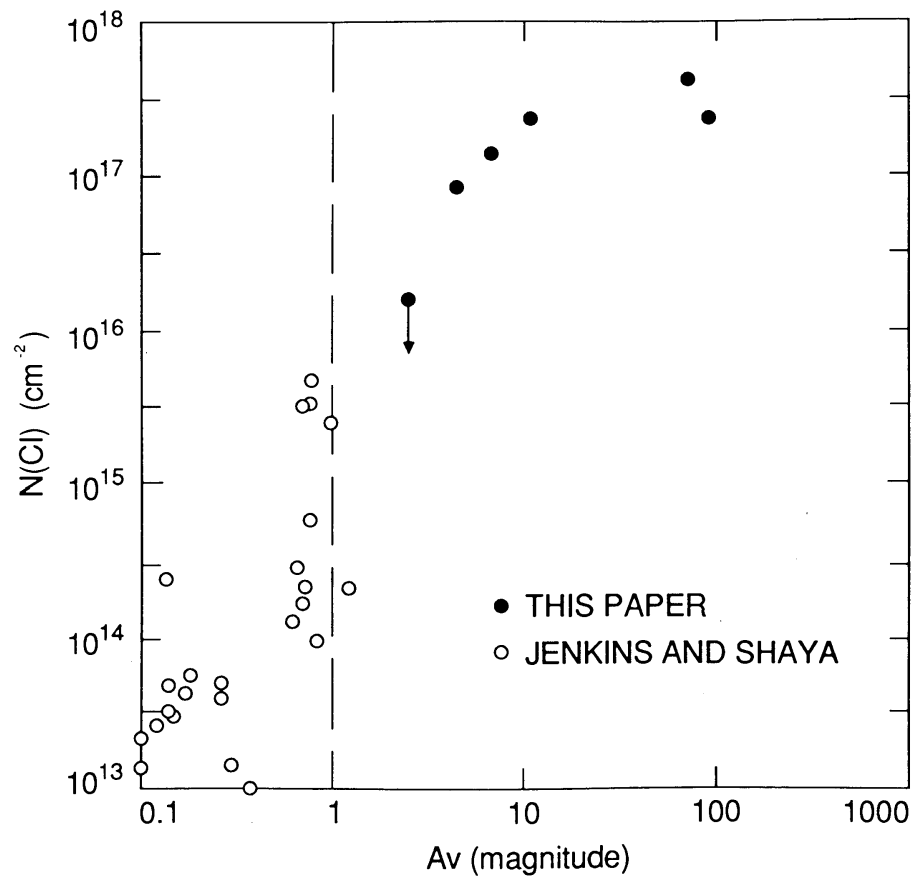

FIG. 3.-The column density of $C_{1}$ as a function of visual extinction. The open circles are taken from data of Jenkins and Shaya (1979). $N\left(C_{1}\right)$ is observed to increase with $A_{V}$ until the visual extinction reaches $100 \mathrm{mag}$. The value of $N\left(\mathrm{C}_{\mathrm{I}}\right)$ at $100 \mathrm{mag}$ is a lower limit since the $\mathrm{C}_{\mathrm{I}}$ line appears to be strongly self-absorbed. 


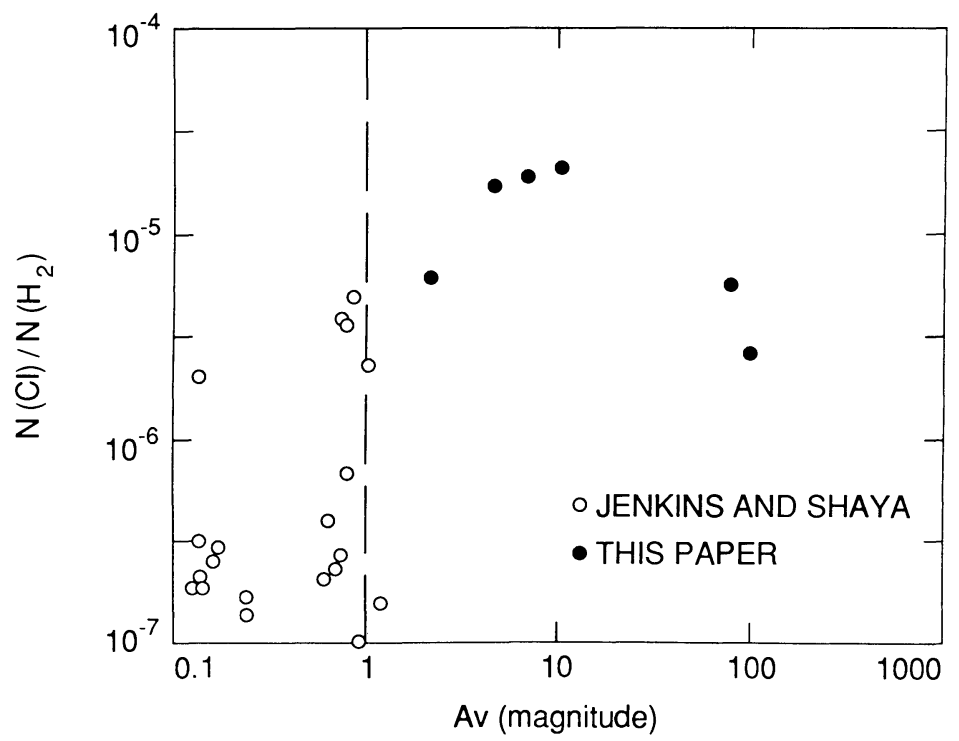

FIG. 4.-The column-averaged fractional abundance of $\mathrm{C} \mathrm{I}, N(\mathrm{C} \mathrm{I}) / N\left(\mathrm{H}_{2}\right)$, plotted as a function of visual extinction. The open circles are taken from data of

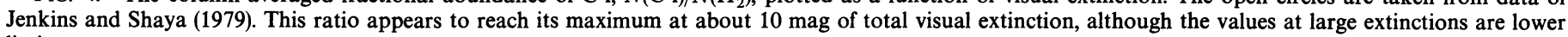
limits.

where the usual conversion, $N\left(\mathrm{H}_{2}\right) / A_{V}=0.94 \times 10^{21}$ has been assumed (Bohlin, Savage, and Drake 1978). The columnaveraged fractional abundance first increases with $A_{V}$ to about $2.2 \times 10^{-5}$ at $4-11 \mathrm{mag}$, then appears to decrease at $100 \mathrm{mag}$. This implies that $\mathrm{C}_{\mathrm{I}}$ is probably never the primary reservoir for gaseous carbon, even at cloud edges. $\mathrm{CO}$ is observed to have a fractional abundance of $8.5 \times 10^{-5}$ in dark cloud cores (FLW), a factor of 4 greater than the maximum fractional abundance of $\mathrm{C}$ I. Similarly, in the diffuse interstellar medium the fractional abundance of $\mathrm{C}$ II is about $1 \times 10^{-4}$ (Morton $e t$ al. 1973).

Since the above values are column averaged, the local fractional abundances may actually be quite different. If we model the path toward the high-excitation sources as passing through two regions, the first a shell with $X(\mathrm{C} \mathrm{I})=2.2 \times 10^{-5}$, as is seen toward the intermediate extinction sources, and the center a core with no $C_{I}$, then the thickness in radius of the $C_{I}$ rich shell must be at least 10 mag for Oph A and 6 mag for Oph B.

\section{d) Quantitative Comparisons of $\mathrm{C} \mathrm{I}$ and $\mathrm{CO}$}

To facilitate comparison, $\mathrm{CO}$ abundances are calculated using the same assumptions as $N(\mathrm{C} \mathrm{I})$. The $N(\mathrm{CO})$ cannot be derived directly from ${ }^{12} \mathrm{CO}$ measurements because ${ }^{12} \mathrm{CO}$ emission is usually very strongly saturated. The less abundant isotopic species, ${ }^{13} \mathrm{CO}$ and $\mathrm{C}^{18} \mathrm{O}$, scaled by appropriate isotopic ratios, are used instead. The problems of this scaling have been well documented in the literature (cf. FLW and references cited therein). The ${ }^{13} \mathrm{CO}$ emission may be saturated at moderate depths within clouds and the ratio of ${ }^{13} \mathrm{CO}$ to ${ }^{12} \mathrm{CO}$ may be enhanced by chemical fractionation or decreased by isotopic selective photodestruction. The less abundant isotope, $\mathrm{C}^{18} \mathrm{O}$, is less affected by saturation and is not subject to fractionation but its abundance can be decreased by selective photodestruction. However, the $\mathrm{C}_{\mathrm{I}}$ lines appear to be quite similar to those of ${ }^{13} \mathrm{CO}$ in both intensity and shape so that $N(\mathrm{C} \mathrm{I}) /$ $N\left({ }^{13} \mathrm{CO}\right)$ may be more reliably determined than $N(\mathrm{C} \mathrm{I}) /$ $N\left(\mathrm{C}^{18} \mathrm{O}\right)$. The column densities for ${ }^{13} \mathrm{CO}$ and $\mathrm{C}^{18} \mathrm{O}$, given in
Table 3, were derived by FLW for the four lower extinction sources, while those for Oph A and Oph B were calculated from our observations by the method described by FLW.

To estimate the value of the ratio $N(\mathrm{C} \mathrm{I}) / N(\mathrm{CO})$ we multiply $N(\mathrm{C} \mathrm{I}) / N\left({ }^{13} \mathrm{CO}\right)$ and $N(\mathrm{C} \mathrm{I}) / N\left(\mathrm{C}^{18} \mathrm{O}\right)$ by 75 and 500 respectively, the interstellar isotopic ratios. These scaled ratios are shown in Figure 5 as a function of visual extinction along with the results from Federman et al. at lower $A_{V}$. The columnaveraged ratio of $\mathrm{C}$ I to $\mathrm{CO}$ is observed to decrease with $A_{V}$ from about 10 at low extinctions, to 1 at about $2 \mathrm{mag}$, and greater than $\sim 0.03$ at 100 mag.

\section{DISCUSSION}

The Ophiuchus molecular cloud complex is a region of active star formation (Wilking and Lada 1983; Elias 1978). The newly formed young stars enhance the UV radiation field over the average in the interstellar medium. As can be seen in Table 3 (and also as discussed by FLW), the ${ }^{13} \mathrm{CO}$ emission appears to be enhanced with respect to the $\mathrm{C}^{18} \mathrm{O}$ emission, presumably by chemical fractionation, much farther into the cloud than typical of most interstellar clouds. Such an effect is consistent with a larger than average radiation field. Therefore, the UV field in the Ophiuchus molecular cloud complex is probably somewhat greater than the average interstellar field, but it is not likely to be as intense as that in high-mass star-forming regions.

An embedded radio continuum source BZ 4 (Brown and Zuckerman 1975) is located $2^{\prime} \mathrm{E}$ of our Oph A position. It is observed to emit carbon recombination line (Brown et al. 1974) and far-infrared continuum radiation (Harvey, Campbell, and Hoffmann 1979). The radio continuum source FG 12 (Falgarone and Gilmore 1981) is observed in the vicinity of Oph $\mathbf{B}$ but there is no $\mathrm{C}$ II recombination emission or farinfrared continuum radiation present. These factors indicate local variations in the radiation field which could contribute in part to the higher $N(\mathrm{C}$ I) observed toward Oph A than toward Oph B. Self-absorption of the $C_{\mathrm{I}}$ line toward Oph B and its 


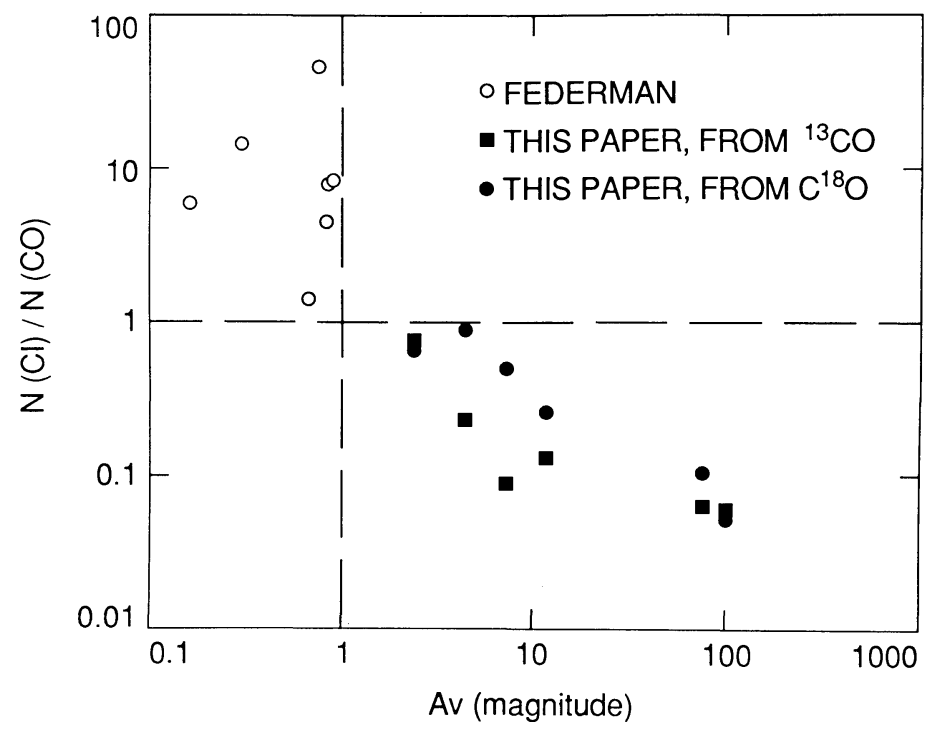

FIG. 5. - The ratio, $N(\mathrm{C} \mathrm{I}) / N(\mathrm{CO})$ derived from both $N\left({ }^{13} \mathrm{CO}\right)$ and $N\left(\mathrm{C}^{18} \mathrm{O}\right)$, as a function of visual extinction, $A_{V}$. The open circles are taken from data of Federman et al. (1980). The ratio steadily decreases toward high-extinction regions. It appears to pass through 1 at an $A_{V}$ of about 2 mag.

location in a core with a greater $A_{V}$ are also contributing factors.

For the observations presented here, we have measured column densities that yield an average of the abundance along the line of sight. In contrast, most theoretical calculations give the fractional abundance as a function of depth into the cloud as parameterized by visual extinction. For the four low and intermediate extinction sources, R17, R48, R38, and R35, the visual extinctions are measured to field stars lying behind the molecular material. Since these are random lines of sight, the density is probably not strongly peaked at some position along them. Therefore, an upper limit of the $A_{V}$ to use for comparison with theoretical models can be derived by assuming that all the material toward these positions is distributed uniformly along the line of sight. In comparisons of the observations to the models, the observed $A_{Y}$ 's should be halved to account for the two outer edges of the cloud.

\section{a) Comparison with Models}

We shall discuss our observations in terms of two theoretical calculations: that of van Dishoeck and Black (1988), a timeindependent model, and the time-dependent model of Tarafdar et al. (1985).

In the interstellar medium, carbon is found in several different forms. In the gaseous state it exists as $\mathrm{C}$ II, C I, CO, and various other carbon-bearing molecules. Outside dense molecular clouds, where the interstellar radiation field is intense, carbon is found primarily in the form of $\mathrm{C}$ II (e.g., Morton et al. 1973). Near the edges of a cloud, C II recombines with electrons to form $\mathrm{C}$ I. The $\mathrm{C}$ I can be hydrogenated to make the species $\mathrm{CH}, \mathrm{CH}_{2}$, and $\mathrm{CH}_{3}$ which, in turn, react with oxygen and oxygen-bearing molecules to form $\mathrm{CO}$. The transition from $\mathrm{C}_{\text {II }}$ to $\mathrm{C}_{\mathrm{I}}$ depends on the ratio of the production of $\mathrm{C}_{\mathrm{I}}$ via recombination of $\mathrm{C}$ II with electrons, to its destruction by UV photoionization. The depth into the cloud at which this transition occurs depends in large part on the magnitude of the UV radiation field. The conversion of $\mathrm{C} \mathrm{I}$ into $\mathrm{CO}$ also depends on the local conditions.
The van Dishoeck and Black model treats a large range of clouds, from diffuse clouds where UV photoprocesses dominate, through translucent clouds (up to 13 mag extinction) where photoprocesses are still important but ion-molecular processes also begin to have a significant effect, to dense photodissociation regions. They have studied the effects of the variations in the external UV radiation field and in the depletion of gas-phase carbon on the derived $\mathrm{C}$ I, $\mathrm{C}$ II, and $\mathrm{CO}$ abundances. In their standard translucent cloud model, with the UV field equal to the mean interstellar field (Draine 1978) and the gaseous carbon depleted to 0.4 of the solar system value, the attenuation of UV photons by grains is large enough so that the production of $\mathrm{C} I$ becomes efficient at visual extinctions of about 0.7 mag into the cloud. The calculated $\mathrm{C}_{\mathrm{I}}$ and $\mathrm{CO}$ abundances are equal at about $2 \mathrm{mag}$, the fractional abundance of $\mathrm{C}$ I peaks between visual extinctions of 2 and $3 \mathrm{mag}$, and gaseous carbon is almost all in the form of $\mathrm{CO}$ at visual extinctions greater than about 5 mag. With a UV field a factor of 10 greater than the mean interstellar field, the $C_{I}$ is produced farther into the cloud, its abundance equals that of $\mathrm{CO}$ at 4 mag, and its fractional abundance peaks between 4 and 5 mag at a slightly larger value than the standard model. If the gaseous carbon depletion is 0.1 , the spatial distribution of $\mathrm{C}_{\mathrm{I}}$ is quite similar to the standard model but its abundance is lower by a factor of about 10 .

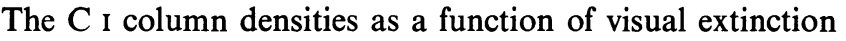
calculated for their standard translucent cloud model, a model with the UV field enhanced by 10 , and a model with the total gaseous carbon abundance depleted to 0.1 of the solar system value are shown in Figure $6 a$ along with our observations. For the high $A_{V}$ regions, particularly Oph A, an enhanced UV field is required to explain the data, while at low extinctions more carbon depletion is required. Since, as mentioned earlier, Oph A contains an embedded radio continuum and carbon recombination line source, it is reasonable that the local UV radiation field is higher by an order of magnitude for that one source. In contrast, Oph B emits only weak radio continuum and no known carbon recombination line radiation, and 


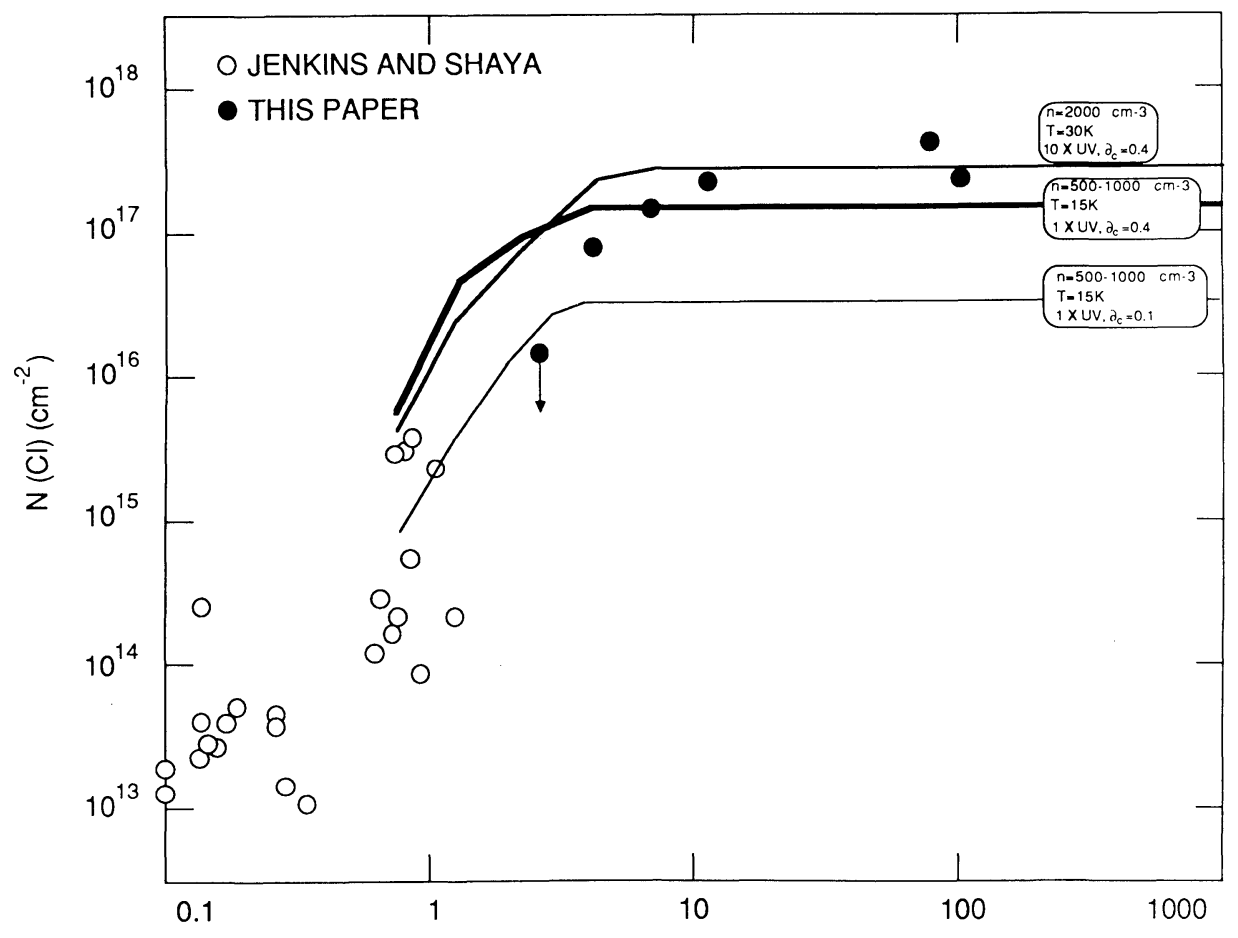

Av (magnitude)

Fig. $6 a$

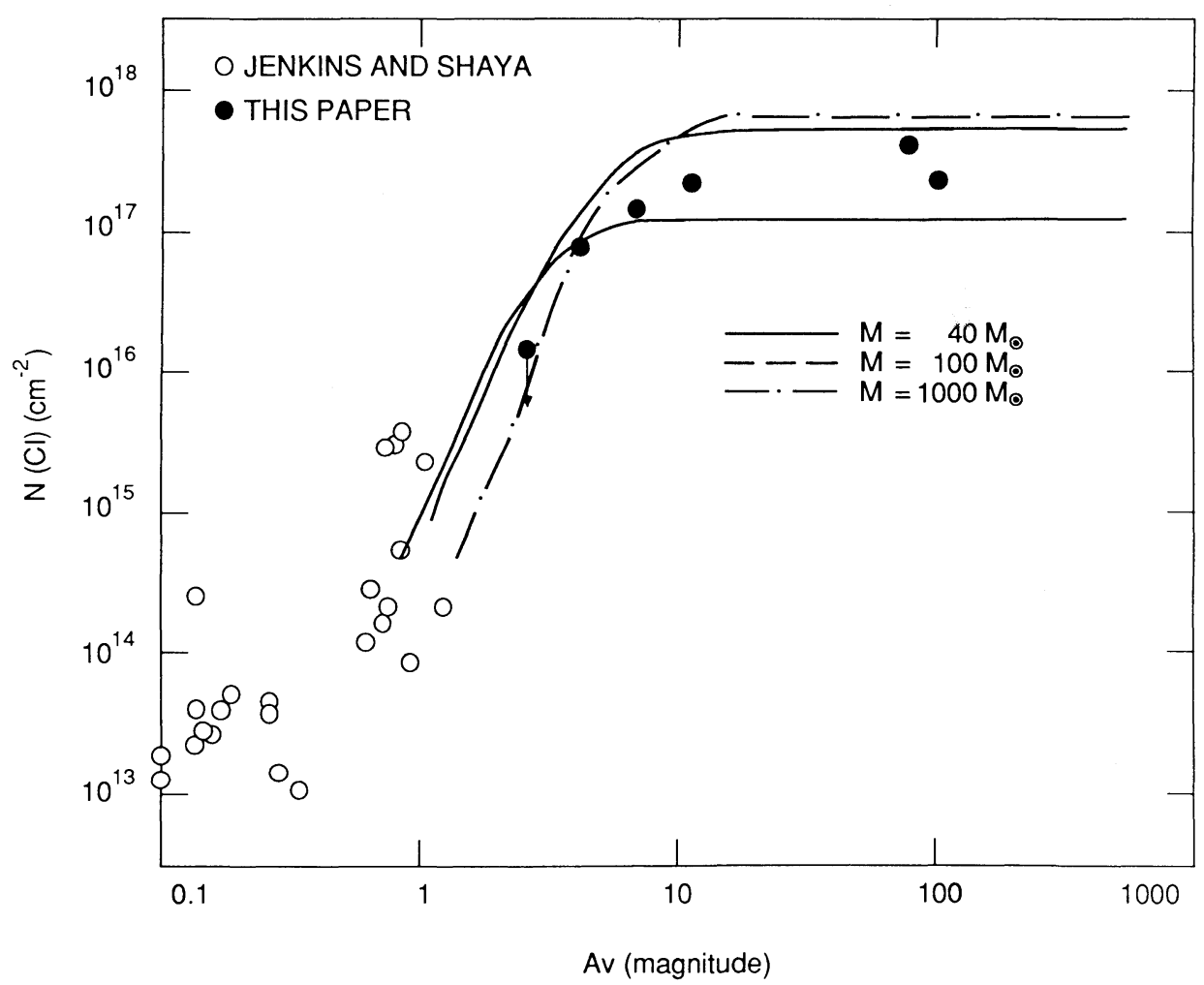

Fig. $6 b$

FIG. 6. - The column density of $\mathrm{C}_{\mathrm{I}}$ as a function of visual extinction superposed on (a) the predictions of van Dishoeck and Black (1988). In (a) the heavy curve shows the results of their standard model with the UV field equal to the average interstellar field and a depletion of gaseous carbon of 0.4 ; the lower light curve shows the results for a gaseous carbon depletion factor of 0.1 ; the upper light curve shows the results for a UV field enhanced by a factor of 10 ; and $(b)$ the calculations of Tarafdar et al. (1985). The visual extinction corresponds to depth into the clouds, hence the observations are plotted at half their observed $A_{V}$ (see text for discussion). 
appears to have less need for an enhanced radiation field in the models (but we emphasize again that we may have underestimated the carbon abundance because of line self-absorption).

Several time-dependent chemical (Graedel, Langer, and Frerking 1982) and physical-chemical (Gerola and Glassgold 1978; Tarafdar et al. 1985) models of evolving clouds have been made. At cloud ages greater than a few times $10^{6} \mathrm{yr}$ all the $\mathrm{C} \mathrm{I}$ has been converted into $\mathrm{CO}$, but at earlier times the $\mathrm{C}$ I abundance can be quite large, even larger than that of $\mathrm{CO}$. For instance, in the calculations by Graedel, Langer, and Frerking, $X\left(\mathrm{C}\right.$ I) exceeds $X(\mathrm{CO})$ for ages less than about $3 \times 10^{5} \mathrm{yr}$.

The calculations of Tarafdar et al. (1985) incorporated cloud collapse as well as time-dependent chemical evolution. We present the theoretical $N(\mathrm{C}$ I) as a function of visual extinction through the center of Tarafdar et al.'s model clouds (taken from their Fig. 7) as they evolve from the diffuse to the dense state in Figure $6 b$. Superposed on their models are our observed data points. The total column density in this calculation is large because most of the cloud lifetime is spent in the diffuse state where gaseous carbon is found primarily in $\mathrm{C} \mathrm{I}$. However, the model calculations were not carried out beyond $4 \times 10^{6}$ yr. Since the Ophiuchus molecular cloud complex is a region of active star formation, it is possibly considerably older than this.

\section{b) Other Possible Sources of Large C I Abundances}

Stutzki et al. (1988) and Genzel et al. (1988), inspired by observations of $\mathrm{C}$ II widely distributed throughout the M17 molecular cloud, have suggested that clumpy structures within molecular clouds allow UV radiation, either externally or internally generated, to penetrate these clouds forming $C_{I}$ and C II interfaces on the surfaces of clumps throughout.

An alternative explanation for the large observed $\mathrm{C}$ I abundance, proposed by Langer et al. (1984), is that carbon is more abundant than oxygen in the gaseous phase within dense clouds. Calculations show that the $\mathrm{C}_{\mathrm{I}}$ fractional abundance is particularly sensitive to the total gaseous carbon abundance. For instance, Glassgold, Huggins, and Langer (1985) find that if the total carbon abundance is increased by a factor of 2 , then the $C_{\text {I }}$ fractional abundance will increase by a factor of 4 . Most models assume solar system abundance ratios, with oxygen more abundant than carbon by a factor of 2 . In this case, at high $A_{V}$ all the carbon is tied up in $\mathrm{CO}$, leaving a reservoir of excess oxygen. If the reverse were true, after all the oxygen were converted into $\mathrm{CO}$ a reservoir of carbon would remain. This result is independent of cloud physical or chemical evolution. Tarafdar, Prasad, and Huntress (1983) found that in diffuse clouds the ratio of $\mathrm{C}$ to $\mathrm{O}$ appears to vary considerably, from $<1$ to $>1$. Measurements of the primary oxygen-bearing species, $\mathrm{O}_{2}$ and $\mathrm{H}_{2} \mathrm{O}$, in dense clouds would test this hypothesis. Searches for $\mathrm{O}_{2}$, in the isotopic form $\mathrm{O}^{18} \mathrm{O}$, in the interstellar medium have not been successful (Liszt and Vanden Bout 1985; Goldsmith et al. 1985) and $\mathrm{H}_{2} \mathrm{O}$ has only been observed in shocked or extremely excited regions but not in typical molecular clouds (Waters et al. 1980; Phillips, Kwan, and Huggins 1980; Frerking and Kuiper 1988).

Yet another possibility would be to invoke another pool of carbon not considered in the available gas-phase chemistry models. A source of carbon at high $A_{V}$ is required. The fractional abundance of $\mathrm{CO}$ above $10 \mathrm{mag}$ of extinction is about $0.85 \times 10^{-4}$ while the cosmic fractional abundance of carbon $[N(\mathrm{C}) / N(2 \mathrm{H})]$ is $6.6 \times 10^{-4}$ (Allen 1973), a factor of nearly 8 larger. Most of this residual carbon is presumably locked in grains. If there were some mechanism that removed just a few percent of the carbon from grains at high $A_{V}$, then sufficient free carbon would be available to explain the observations.

Since the Ophiuchus molecular cloud complex is a region of active star formation, the energetics associated with young stars can serve as the source both of turbulence and internal radiation fields. Mixing of material from cloud edge regions with the core material could enhance the depths to which $\mathrm{C} I$ is found within clouds. Also, since internal sources of UV radiation are available, perhaps the starting time for chemistry is reset locally each time a star is formed, enhancing the $\mathrm{C} \mathrm{I}_{\mathrm{I}}$ abundance near young stars.

\section{SUMMARY}

We have observed the $492 \mathrm{GHz}$ line of $\mathrm{C}$ I toward six positions in the Ophiuchus molecular cloud complex for which accurate determination of the visual extinction is available. We have revised the $C_{I}$ line frequency to $492160.7 \pm 0.1 \mathrm{MHz}$ based on these observations.

We find that $\mathrm{C} \mathrm{I}$ is less abundant in the deep cores of clouds at very high visual extinctions than it is at intermediate $A_{V}$ 's. However, its fractional abundance remains high for at least 10 mag into a cloud. The column-averaged fractional abundance of $C \mathrm{I}$ is less than $\sim 6 \times 10^{-6}$ at $2.6 \mathrm{mag}, 2.2 \times 10^{-5}$ between 4 and $11 \mathrm{mag}$, and greater than $\sim 2-5 \times 10^{-6}$ at $100 \mathrm{mag}$. The column-averaged abundance ratio of $\mathrm{C}$ I to $\mathrm{CO}$ is slightly less than 1 from 2 to $4 \mathrm{mag}$, and decreases by a factor of $\sim 20$ at 100 mag.

The $C_{\text {I }}$ abundances for visual extinctions less than about 5 mag can be explained with standard gas-phase ion-molecular chemical theory after taking into account variations in density and temperature. The abundances at $A_{V}$ greater than $5 \mathrm{mag}$, however, require some other explanation.

We have compared our results with two published theories which attempt to explain the $\mathrm{C}$ I column densities observed at intermediate and high $A_{V}$. One is the equilibrium model of van Dishoeck and Black (1988) incorporating variations in the UV radiation field and in the amount of gas-phase carbon depletion. The second is the chemical-physical evolution calculation of Tarafdar et al. (1985). In this model the C $\mathrm{I}$ abundance is enhanced because clouds spend much of their lifetime in a diffuse state where the rate of $\mathrm{CO}$ formation is very slow.

Several other mechanisms have been suggested to explain the observations. Stutzki et al. (1988) and Genzel et al. (1988) have suggested that clumpy structures within molecular clouds allow penetration of UV radiation into the clouds, forming large $\mathrm{C}$ I and $\mathrm{C}$ II abundances throughout. An abundance ratio of $\mathrm{C}$ to $\mathrm{O}$ higher than the solar system ratio also explains the observations without resorting to time-dependent scenarios; however, it has not been confirmed observationally in dense clouds. Another possibility is that at large $A_{V}$ carbon is released into the gaseous state from grains, perhaps through heating in star-forming cores. Mixing of material in the edge regions with the core region is also possible. Young, active stars can serve as a source of turbulence and also of internal radiation fields, perhaps resetting the chemical clock locally each time a star is formed.

We would like to thank W. D. Langer for many useful discussions, E. van Dishoeck for a preprint of her work, and the crew of the NASA Kuiper Airborne Observatory for their help and cooperation. We are grateful to C. A. Beichman and N. J. 
Evans for their help in obtaining the $\mathrm{C}_{\mathrm{I}}$ and $\mathrm{CO}$ spectra, respectively. The airborne observations were supported by NASA grant NAG 2-1 to Caltech. Partial support for M. A.
Frerking was supplied by the Jet Propulsion Laboratory, California Institute of Technology, under contract with the National Aeronautics and Space Administration.
Allen, C. W. 1973, Astrophysical Quantities (London: Athlone Press). Bohlin, R. C., Savage, B. D., and Drake, J. F. 1978, Ap. J., 224, 132.

Boland, W., and de Jong, T. 1984, Ap. J., 134, 87.

Brown, E. R., Keene, J., and Phillips, T. G. 1985, Internat. J. Infrared Millimeter Waves, 6,1121 .

Brown, R. L., Gammon, R. H., Knapp, G. R., and Balick, B. 1974, Ap. J., 192, 607.

Brown, R. L., and Zuckerman, B. 1975, Ap. J. (Letters), 202, L125.

Cooksy, A. L., Saykally, R. J., Brown, J. M., and Evenson, K. M. 1986, Ap. J., 309, 828 .

de Jong, T., Dalgarno, A., and Boland, W. 1980, Astr. Ap., 91, 68.

Draine, B. T. 1978, Ap. J. Suppl., 36, 595.

Elias, J. H. 1978, Ap. J., 224, 453.

Falgarone, E., and Gilmore, W. 1981, Astr. Ap., 95, 32

Federman, S. R., Glassgold, A. E., Jenkins, E. B., and Shaya, E. J. 1980, Ap. J., 242, 545 .

Frerking, M. A., and Kuiper, T. 1988, in Interstellar Matter, ed. J. M. Moran and P. T. P. Ho (New York: Gordon \& Breach), p. 185.

Frerking, M. A., Langer, W. D., and Wilson, R. W. 1982, Ap. J., 262, 590 (FLW).

Genzel, R., Harris, A. I., Jaffe, D. T., and Stutzki, J. 1988, Ap. J., 332, 1049.

Gerola, H., and Glassgold, A. E. 1978, Ap. J. Suppl., 37, 1.

Glassgold, A. E., Huggins, P. J., and Langer, W. D. 1985, Ap. J., 290, 615.

Goldsmith, P. F., Snell, R. L., Erickson, N. R., Dickman, R. L., Schloerb, F. P., and Irvine, W. M. 1985, Ap. J., 289, 613.

Graedel, T. E., Langer, W. D., and Frerking, M. A. 1982, Ap. J. Suppl., 48, 321.

Harvey, P. M., Campbell, M. F., and Hoffmann, W. 1979, Ap. J., 228, 445.

Iglesias, E. 1977, Ap. J., 218, 697.

Jenkins, E. B., and Shaya, E. J. 1979, Ap. J., 231, 55.

Keene, J., Blake, G. A., Phillips, T. G., Huggins, P. J., and Beichman, C. A. 1985, Ap. J., 299, 967.

\section{REFERENCES}

Kutner, M. L., and Ulich, B. L. 1981, Ap. J., 250, 341

Langer, W. D. 1976, Ap. J., 206, 699.

Langer, W. D., Graedel, T. E., Frerking, M. A., and Armentrout, P. B., 1984, Ap. J., 277, 581.

Liszt, H. S., and Vanden Bout, P. A. 1985, Ap. J., 291, 178.

Mitchell, G. F., Ginsburg, J. L., and Kuntz, P. J. 1978, Ap. J. Suppl., 38, 39.

Morton, D. C., Drake, J. F., Jenkins, E. B., Rogerson, J. B., Spitzer, L., and York, D. G. 1973, Ap. J. (Letters), 181, L103.

Nussbaumer, H. 1971, Ap. J., 166, 411.

Nussbaumer, H., and Rusca, C. 1979, Astr. Ap., 72, 129.

Phillips, T. G., and Huggins, P. J. 1981, Ap. J., 251, 533

Phillips, T. G., Kwan, J., and Huggins, P. J. 1980, in Interstellar Molecules, ed. B. H. Andrew (Dordrecht: Reidel), p. 21.

Prasad, S. S., and Huntress, W. T., Jr. 1980, Ap. J., 239, 151

Saykally, R. J., and Evenson, K. 1980, Ap. J. (Letters), 238, L107.

Stutzki, J., Stacey, G. J., Genzel, R., Harris, A. I., Jaffe, D. T., and Lugten, J. B. 1988, Ap. J., 332, 379.

Tarafdar, S. P., Prasad, S. S., and Huntress, W. T., Jr. 1983, Ap. J., 267, 156

Tarafdar, S. P., Prasad, S. S., Huntress, W. T., Jr., Villere, K. R., and Black, D. C. 1985, Ap. J., 289, 220.

Tielens, A G. G. M and Hollenbach, D. 1985, Ap J. 291, 722.

van Dishoeck, E. F., and Black, J. H. 1988, Ap. J., 334, 771.

Waters, J. W., Gustincic, J. J., Kakar, R. K., Kuiper, T. B. H., Roscoe, H. K., Swanson, P. N., Ridriguez Kuiper, E. N., Kerr, A. R., and Thaddeus, P. 1980, Ap. J., 235, 57.

Wilking, B. A., and Lada, C. J. 1983, Ap. J., 274, 698.

Wootten, A., Phillips, T. G., Beichman, C. A., and Frerking, M. A. 1982, Ap. J. (Letters), 256, L5.

G. A. Blake: California Institute of Technology, 170-25, Pasadena, CA 91125

M. A. Frerking: Jet Propulsion Laboratory, 168-314, Pasadena, CA 91109

J. KeEne and T. G. PhILliPS: California Institute of Technology, 320-47, Pasadena, CA 91125 